Article

\title{
Spectrum and Energy Efficiency of Uplink Massive MIMO System with D2D Underlay
}

\author{
Xinhua Wang \\ College of Automation and Electrical Engineering, Qingdao University, Qingdao 266071, China; \\ xhwang@qdu.edu.cn; Tel.: +86-159-6982-0800 \\ Academic Editors: Jaap Van de Beek, Luca Chiaraviglio, William Liu and Filip Idzikowski \\ Received: 7 March 2017; Accepted: 12 April 2017; Published: 13 April 2017
}

\begin{abstract}
In this paper, both the spectrum efficiency (SE) and the energy efficiency (EE) are investigated for an uplink massive multiple-input multiple-output (MIMO) system coexisting with an underlay device-to-device (D2D) system. The outage probability and the achievable rates of the cellular user equipments (CUEs) and the D2D link are derived in closed-form, respectively. Constrained by the SE of the D2D link and the CUEs, the EE of the massive MIMO system is maximized by jointly optimizing the transmit power of CUEs and the number of BS antennas. An algorithm with low complexity is proposed to solve the optimization problem. Performance results are provided to validate our derived closed-from results and verify the efficiency of our proposed scheme.
\end{abstract}

Keywords: device-to-device; energy efficiency; massive MIMO; spectrum efficiency; outage probability

\section{Introduction}

In the past few years, the Fourth Generation (4G) of mobile telecommunication has been significantly changing and improving our lives. Meanwhile, the exponentially increasing rate requirements throw down the gauntlet to the latest standard for the mobile telecommunication. Nowadays, research interest has already been drawn on the the Fifth Generation (5G) of mobile telecommunication which can achieve substantially higher throughput than 4G. Massive multiple-input multiple-output (MIMO) and DEVICE-to-device (D2D) communication are two promising candidate technologies of 5G. Massive MIMO employing a few hundreds of antennas at the BS can achieve a higher data rate through focusing the energy into a narrow beam towards the receiver. $\mathrm{D} 2 \mathrm{D}$ is another emerging technology to meet the high-rate data requirements due to the short-range direct transmission without going through the base station (BS).

\subsection{Related Works}

Compared with the traditional MIMO system, the large-scale antennas can bring in the higher spectrum efficiency (SE) and energy efficiency (EE) [1-3]. Lower capacity bounds for linear detection are derived from the uplink massive MIMO systems [3]. Conjugate and Zero-Forcing precoders are compare in terms of SE and EE for the downlink of massive MIMO systems [4]. Nguyen et al. compared massive MIMO and small cells in terms of user-average SE by using stochastic geometry theory [5]. Masini et al. analyzed MC-CDMA systems adopting an adaptive detection technique based on threshold orthogonality restoring combining $[6,7]$. Wen et al. derived the asymptotic sum-rate of the multiuser (MU) MIMO uplink channel in the large-system regime [8].

D2D underlaying cellular network $(\mathrm{CN})$ is regarded as a means to increase the data rate, and decrease the load of $\mathrm{CNs}[9,10]$. However, the coexistence of the D2D and the $\mathrm{CN}$ causes the mutual interference. To combat the interference from the D2D to the $\mathrm{CN}$, Doppler et al. proposed a mechanism 
to limit the maximum transmit power of the D2D transmitter [11]. However, the interference from the $\mathrm{CN}$ to the D2D link is not considered. In [12], the cellular user equipments (CUEs) are scheduled for the communication if they are not in the interference-limited area so as not to generate harmful interference to the D2D receiver. Bazzi et al. analyzed the performance of D2D enabling V2V Beaconing [13]. Palombara et al. developed a framework for design and analysis of relay-assisted diversity communications accounting [14].

The combination of massive MIMO and D2D is investigated in [15-17]. Lin et al. adopt the partial zero-forcing receiving technique to cancel the uplink interference from the nearby CUEs and D2D links, and investigate the spectral efficiency with both the perfect and imperfect channel state information (CSI) [15]. The precoding and power allocation are jointly optimized for D2D underlaying massive MIMO multicasting networks [16]. Shalmashi et al. derived average sum rate and EE for massive MIMO system with D2D links underlay [17].

\subsection{Motivation and Contribution}

To guarantee a required achievable rate, with perfect CSI available, Ngo et al. show that the transmit power of the CUEs can be made inversely proportional to the number of antennas in the uplink of massive MIMO systems [3]. Inspired by this fact, we limit the maximum transmit power of the CUEs to suppress the interference from the $\mathrm{CN}$ to the $\mathrm{D} 2 \mathrm{D}$, and then guarantee the $\mathrm{CN}$ performance with the aid of large-scale antennas.

In this paper, we consider an uplink massive MIMO system with D2D underlay. The main contributions of this work are summarized as:

- The probability density function of the signal-to-interference-plus-noise ratio (SINR) at the CUEs is approximated in closed-form.

- The outage probabilities and the achievable rates of the massive MIMO system and the D2D link are derived in closed-form.

- Constrained by the SE of the D2D link and CUEs, the EE of the massive MIMO system is maximized by jointly optimizing the transmit power of CUEs and the number of BS antennas.

\section{System Model}

We consider a massive MIMO system with a D2D link underlay as shown in Figure 1, which consists of a BS with $N$ antennas, $K$ CUEs, and one D2D link. Each CUE or D2D user is equipped with a single omnidirectional antenna. The CUEs transmit signal to the BS using the same time-frequency resource as the D2D link.

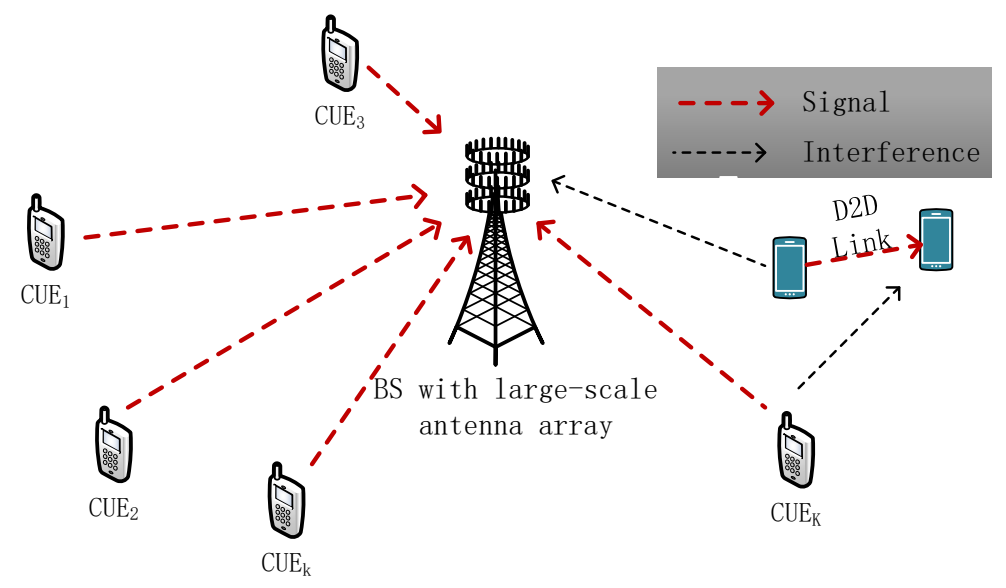

Figure 1. System Model.

Let $\sqrt{\beta_{c k}} \mathbf{h}_{c k}$ denote the channel vector from the $k$-th CUE to the BS, where $\beta_{c k}$ and $\mathbf{h}_{c k} \sim \mathcal{C N}(0, \mathbf{I})$ represent the large-scale fading (path-loss) and the small-scale fading, respectively. Similarly, $\sqrt{\beta_{d}} \mathbf{h}_{d}$ 
denotes the channel vector from the D2D transmitter to the BS. $\sqrt{\alpha_{c k}} g_{c k}$ and $\sqrt{\alpha_{d}} g_{d}$ represent the channel fading from the $k$-th CUE and the D2D transmitter to the D2D receiver, respectively.

Similarly to [3], the channel vectors from the CUEs to the BS are assumed to be available at the BS, and the maximum ratio combining (MRC) technique is adopted for the data reception. The detected signal of the $k$-th CUE after the linear processing can be given as

$$
r_{c k}=\sqrt{P_{c} \beta_{c k}} \mathbf{h}_{c k}^{H} \mathbf{h}_{c k} s_{c k}+\sum_{j \neq k}^{K} \sqrt{P_{c} \beta_{c j}} \mathbf{h}_{c k}^{H} \mathbf{h}_{c j} s_{c j}+\sqrt{P_{d} \beta_{d}} \mathbf{h}_{c k}^{H} \mathbf{h}_{d} s_{d}+\mathbf{h}_{c k}^{H} \mathbf{n},
$$

where $P_{\mathrm{c}}$ and $P_{\mathrm{d}}$ represent the transmit powers of the CUEs and the D2D transmitter, respectively. $\mathbf{n} \sim \mathcal{C N}\left(0, \sigma^{2} \mathbf{I}\right)$ is an additive white Gaussian noise (AGWN) vector at the BS. In addition, $s_{d}$ and $s_{c k}$ denote respectively the normalized signal sent by the D2D transmitter and the $k$-th CUE, i.e., $\mathbb{E}\left[\left|s_{d}\right|^{2}\right]=\mathbb{E}\left[\left|s_{c k}\right|^{2}\right]=1$. The SINR at the $k$-th CUE is denoted as $\gamma_{c k}$ and given as

$$
\gamma_{c k}=\frac{S_{c k}}{I_{c k}+\sigma^{2}}
$$

where $I_{c k}=P_{\mathrm{d}} \beta_{d}\left|\frac{\mathbf{h}_{c k}^{H}}{\left\|\mathbf{h}_{c k}\right\|} \mathbf{h}_{d}\right|^{2}+\sum_{j \neq k}^{K} P_{\mathrm{c}} \beta_{c j}\left|\frac{\mathbf{h}_{c k}^{H}}{\left\|\mathbf{h}_{c k}\right\|} \mathbf{h}_{c j}\right|^{2}$ and $S_{c k}=P_{c} \beta_{c k}\left\|\mathbf{h}_{c k}\right\|^{2}$. The average achievable rate of the $k$-th CUE is

$$
\mathcal{R}_{c k}=\mathbb{E}\left[\log _{2}\left(1+\gamma_{c k}\right)\right] .
$$

The received signal at the D2D receiver can be given as

$$
y_{d}=\sqrt{P_{\mathrm{d}} \alpha_{d}} g_{d} s_{d}+\sum_{k=1}^{K} \sqrt{P_{\mathrm{c}} \alpha_{c k}} g_{c k} s_{c k}+n_{d}
$$

where $n_{d} \sim \mathcal{C N}\left(0, \sigma_{d}^{2}\right)$ is the AGWN at the D2D receiver. The SINR at the D2D receiver is

$$
\gamma_{d}=\frac{S_{d}}{I_{d}+\sigma_{d}^{2}}
$$

where $S_{d}=P_{\mathrm{d}} \alpha_{d}\left|g_{d}\right|^{2}$ and $I_{d}=\sum_{k=1}^{K} P_{c} \alpha_{c k}\left|g_{c k}\right|^{2}$. The average achievable rate of the D2D link is

$$
\mathcal{R}_{d}=\mathbb{E}\left[\log _{2}\left(1+\gamma_{d}\right)\right]
$$

The energy consumption of the massive MIMO system in a normalized time block is

$$
E=K P_{c}+(N+K) P_{\text {cir }}+P_{0}
$$

where $P_{\text {cir }}$ and $P_{0}$ represent the circuit power consumption per RF chain and the basic power consumption of the system, respectively. The EE of the massive MIMO system defined as the ratio between the sum-rate and the energy consumption is given as

$$
\Xi=\frac{\sum_{k=1}^{K} \mathcal{R}_{c k}}{E} .
$$

\section{Spectrum Efficiency Analysis}

In this section, we propose the approximate probability density function (PDF) of the SINR, based on which, we analyze the outage probabilities and the achievable rates for both the D2D and the CUEs by considering the mutual interference between the two systems. 


\subsection{Outage Probability and Achievable Rate of CUEs}

Theorem 1. The PDF of $\gamma_{c k}$ can be accurately approximated as

$$
f_{\gamma_{c k}}(x)=\frac{P_{c} \beta_{c k} N \exp \left(\sigma^{2} / b_{c k}\right)\left(P_{c} \beta_{c k} N / x-\sigma^{2}\right)^{a_{c k}-1}}{\left(b_{c k}\right)^{a_{c k}} \Gamma\left(a_{c k}\right) x^{2} \exp \left(P_{c} \beta_{c k} N / x b_{c k}\right)},
$$

where

$$
a_{c k}=\frac{\left(\sum_{j \neq k}^{K} P_{c} \beta_{c j}+P_{d} \beta_{d}\right)^{2}}{\sum_{j \neq k}^{K} P_{c}^{2} \beta_{c j}^{2}+P_{d}^{2} \beta_{d}^{2}} \text { and } b_{c k}=\frac{\sum_{j \neq k}^{K} P_{c}^{2} \beta_{c j}^{2}+P_{d}^{2} \beta_{d}^{2}}{\sum_{j \neq k}^{K} P_{c} \beta_{c j}+P_{d} \beta_{d}}
$$

and $\Gamma(s)=\int_{0}^{\infty} t^{s-1} e^{-t} \mathrm{~d} t$ is the gamma function.

Proof. See Appendix A.

Given a target SINR $\bar{\gamma}_{c}$, we can obtain the outage probability of the $k$-th CUE according to (A1) and (A2) as,

$$
\mathbb{P}_{c k}^{\text {out }}=\operatorname{Pr}\left\{\gamma_{c k}<\bar{\gamma}_{c}\right\}=\frac{\Gamma\left(a_{c k}, \omega_{c k} / b_{c k}\right)}{\Gamma\left(a_{c k}\right)}
$$

where $\omega_{c k}=P_{c} \beta_{c k} N / \bar{\gamma}_{c}-\sigma^{2}$ and $\Gamma(s, x)=\int_{x}^{\infty} t^{s-1} e^{-t} \mathrm{~d} t$ is the upper incomplete gamma function.

Theorem 2. The achievable rate of the $k$-th CUE can be approximated as

$$
\mathcal{R}_{c k} \approx \frac{\mathcal{J}\left(a_{c k}, b_{c k} / \xi_{c k}\right)-\mathcal{J}\left(a_{c k}, b_{c k} / \sigma^{2}\right)}{\Gamma\left(a_{c k}\right) \ln (2)}+\log _{2}\left(\frac{\xi_{c k}}{\sigma^{2}}\right)
$$

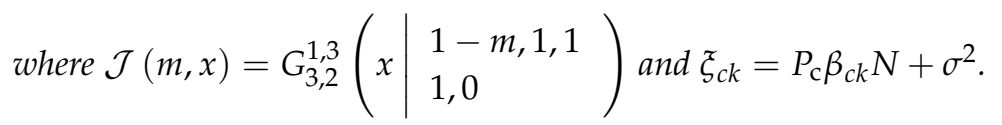

Proof. See Appendix B.

To make the analysis trackable, we also derive the simple lower bound using Jensen's inequality, i.e.,

$$
\tilde{\mathcal{R}}_{c k}=\log _{2}\left(1+P_{c} \beta_{c k} N / \vartheta_{c k}\right)
$$

where $\vartheta_{c k}=P_{\mathrm{d}} \beta_{d}+\sum_{j \neq k}^{K} P_{c} \beta_{c j}+\sigma^{2}$. Note that the achievable rates scales quickly with the number of transmit antennas, and can be made arbitrarily large when $N$ grows without bound.

3.2. Outage Probability and Achievable Rate of the D2D Link

Proposition 1. Given the target SINR $\bar{\gamma}_{d}$ for the D2D link, the outage probability of the D2D link can be obtained as

$$
\mathbb{P}_{d}^{\text {out }}=\operatorname{Pr}\left(\gamma_{d}<\bar{\gamma}_{d}\right)=1-\frac{\exp \left(-\lambda_{d} \bar{\gamma}_{d} \sigma_{d}^{2}\right)}{\left(\lambda_{d} \bar{\gamma}_{d} b_{d}+1\right)^{a_{d}}}
$$

where

$$
\lambda_{d}=\frac{1}{P_{\mathrm{d}} \alpha_{d}}, a_{d}=\frac{\left(\sum_{k=1}^{K} \alpha_{c k}\right)^{2}}{\sum_{k=1}^{K} \alpha_{c k}^{2}}, \text { and } b_{d}=\frac{\sum_{k=1}^{K} \alpha_{c k}^{2}}{\sum_{k=1}^{K} \alpha_{c k}}
$$


Proposition 2. The average achievable rate of the D2D link can be approximated as

$$
\mathcal{R}_{d}=\frac{\mathcal{J}\left(\tilde{a}_{d}, \frac{\tilde{b}_{d}}{\sigma_{d}^{2}}\right)}{\Gamma\left(\tilde{a}_{d}\right) \ln (2)}-\frac{\mathcal{J}\left(a_{d}, \frac{P_{\mathrm{c}} b_{d}}{\sigma_{d}^{2}}\right)}{\Gamma\left(a_{d}\right) \ln (2)},
$$

where

$$
\tilde{a}_{d}=\frac{\left(\sum_{k=1}^{K} P_{\mathrm{c}} \alpha_{c k}+P_{\mathrm{d}} \alpha_{d}\right)^{2}}{\sum_{k=1}^{K} P_{\mathrm{c}}^{2} \alpha_{c k}^{2}+P_{\mathrm{d}}^{2} \alpha_{d}^{2}} \text { and } \tilde{b}_{d}=\frac{\sum_{k=1}^{K} P_{\mathrm{c}}^{2} \alpha_{c k}^{2}+P_{\mathrm{d}}^{2} \alpha_{d}^{2}}{\sum_{k=1}^{K} P_{\mathrm{c}} \alpha_{c k}+P_{\mathrm{d}} \alpha_{d}}
$$

\subsection{Power Management for CUEs}

With the increase of $P_{\mathrm{c}}$, the SE of the $\mathrm{CN}$ can be improved, while the SE of the D2D link is degraded, and vice visa. So, it is interesting to strike a balance between the $\mathrm{CN}$ and the D2D link through the power management for the CUEs. The spectrum performance of the D2D link can be improved effectively by decreasing $P_{\mathrm{c}}$ especially in the high signal-to-noise ratio (SNR) regime. However, the power management of $P_{\mathrm{c}}$ has limited influence on the $\mathrm{CN}$.

Proposition 3. When $P_{c} \rightarrow \infty, \mathbb{P}_{c k}^{\text {out }}$ approaches the lower bound $\hat{\mathbb{P}}_{c k}^{\text {out }}=\Gamma\left(\hat{a}_{c k}, \frac{N \beta_{c k}}{\bar{\gamma}_{c} \hat{b}_{c k}}\right) / \Gamma\left(a_{c k}\right)$, while $\mathcal{R}_{c k}$ approaches the upper bound $\hat{\mathcal{R}}_{c k}$ given as

$$
\hat{\mathcal{R}}_{c k}=\frac{\mathcal{J}\left(\hat{a}_{c k}, \frac{\hat{b}_{c k}}{\beta_{c k} N}\right)}{\Gamma\left(\hat{a}_{c k}\right) \ln (2)}-\frac{\psi\left(\hat{a}_{c k}\right)}{\ln (2)}+\log _{2}\left(\frac{\beta_{c k} N}{\hat{b}_{c k}}\right),
$$

where

$$
\hat{a}_{c k}=\frac{\left(\sum_{j \neq k}^{K} \beta_{c j}\right)^{2}}{\sum_{j \neq k}^{K} \beta_{c j}^{2}}, \hat{b}_{c k}=\frac{\sum_{j \neq k}^{K} \beta_{c j}^{2}}{\sum_{j \neq k}^{K} \beta_{c j}}
$$

and $\psi(x)=\frac{\mathrm{d}}{\mathrm{d} x} \ln \Gamma(x)$

Proof. Let $\rho_{c k}=\sum_{j \neq k}^{K} \beta_{c j}\left|\frac{\mathbf{h}_{c k}^{H}}{\left\|\mathbf{h}_{c k}\right\|} \mathbf{h}_{c j}\right|^{2}$. Similarly to $I_{c k}, \rho_{c k} \sim \operatorname{Gamma}\left(\hat{a}_{c k}, \hat{b}_{c k}\right)$. With the increase of $P_{\mathrm{c}}$, $\mathcal{R}_{c k}$ approaches the following upper bound

$$
\begin{aligned}
& \hat{\mathcal{R}}_{c k}=\mathbb{E}\left[\log _{2}\left(1+\frac{\beta_{c k} N}{\rho_{c k}}\right)\right] \\
& =\int_{0}^{\infty}\left[\log _{2}\left(\beta_{c k} N+\rho_{c k}\right)-\log _{2}\left(\rho_{c k}\right)\right] f\left(\rho_{c k}\right) \mathrm{d} \rho_{c k} \\
& =\frac{l\left(\hat{a}_{c k}, \hat{b}_{c k}, \beta_{c k} N\right)}{\left(\hat{b}_{c k}\right)_{c k} \Gamma\left(\hat{a}_{c k}\right) \ln (2)}-\frac{\psi\left(\hat{a}_{c k}\right)}{\ln (2)}-\log _{2}\left(\hat{b}_{c k}\right) \text {. }
\end{aligned}
$$

where the last step is obtained using ([18] Equation (4.352.1)). After calculating $l\left(\hat{a}_{c k}, \hat{b}_{c k}, \beta_{c k} N\right)$ similarly to Theorem 1, we can conclude the proof.

\section{Energy Efficiency Optimization}

From the last section, we can see that the power management plays a critical role in the SE of the D2D link, while the number of antennas severely influence the SE of the CUEs. The EE with the unit bps $/ \mathrm{Hz} / \mathrm{W}$ is another important metric that should be considered for the green communications. In this section, we aim to maximize the EE $\Xi$ constrained by the SEs of the CUEs and the D2D 
link. To make the optimization problem trackable, we maximized the lower bound of the EE that is $\tilde{\Xi}=\sum_{k=1}^{K} \tilde{\mathcal{R}}_{c k} / E$. The optimization problem can be formulated as

$$
\begin{aligned}
\mathrm{P} 1: \max _{P_{c}, N} & \tilde{\Xi} \\
\text { s.t. } & \mathrm{C} 1: \mathcal{R}_{d} \geq r \\
& \mathrm{C} 2: N P_{\mathrm{c}} \beta_{c k} / \vartheta_{c k} \geq \tilde{\gamma}_{\mathrm{th}}, k=1, \cdots, K \\
& \mathrm{C} 3: N \geq N_{\text {th. }} .
\end{aligned}
$$

$\mathrm{C} 1$ specifies the outage probability and the minimum data rate requirements of the D2D link. C2 specifies the SINR constraints of the CUEs. $N_{\text {th }}$ is a predefined threshold that can guarantee the system operates in the massive MIMO scenario.

To guarantee the performance of the D2D link, the transmit power of CUEs $P_{\mathrm{c}}$ should be limited to less than $P_{\mathrm{c}}^{\max }$, where $P_{\mathrm{c}}^{\max }$ is the solution of the equation $\mathcal{R}_{d}\left(P_{\mathrm{c}}\right)=r$. So, the constraint $\mathrm{C} 1 \mathrm{can}$ be transformed into $P_{\mathrm{c}} \leq P_{\mathrm{c}}^{\max }$. Owing to $\tilde{\gamma}_{\text {th }} \gg 1$, given a value of $P_{\mathrm{c}} \in\left(0, P_{\mathrm{c}}^{\max }\right]$, the optimization problem $\mathrm{P} 1$ can be reformulated as $\mathrm{P} 2$.

$$
\begin{aligned}
\mathrm{P} 2: \max _{N} \bar{\Xi} & =\frac{\sum_{k=1}^{K} \log _{2}\left(P_{c} \beta_{c k} N / \vartheta_{c k}\right)}{N P_{\text {cir }}+K\left(P_{c}+P_{\text {cir }}\right)+P_{0}} \\
\text { s. t. } N & \geq N^{\dagger} .
\end{aligned}
$$

where $N^{+}=\max \left\{\frac{\tilde{\gamma}_{\mathrm{th}} \vartheta_{c k}}{P_{c} \beta_{c k}}, N_{\mathrm{th}}\right\}, k=1, \cdots, K$.

Theorem 3. The optimal number of antennas for the optimization problem P2 is obtained as

$$
N^{\text {opt }}=\max \left\{N^{\dagger},\left\lfloor N^{\S}\right\rceil\right\}
$$

$N^{\S}$ is given as

$$
N^{\S}=\frac{1}{\delta} \exp \left[W\left(\frac{\kappa\left(K P_{c}+K P_{\text {cir }}+P_{0}\right)}{P_{\text {cir }} e}\right)+1\right],
$$

where $W$ is the lambert $W$ function defined as $t=W(t) e^{W(t)}, \delta=P_{\mathrm{c}} \sqrt[K]{\prod_{k=1}^{K} \beta_{c k} / \vartheta_{c k}}$, and $\left\lfloor N^{\S}\right\rceil$ is the one of the two closest integer around $N^{\S}$ that can maximize $\bar{\Xi}$.

Proof. The objective function can be written as

$$
\bar{\Xi}=\frac{\log _{2}(\delta N)}{N P_{\text {cir }}+\left(K P_{c}+K P_{\text {cir }}+P_{0}\right)} .
$$

We can conclude the proof according to the quasi-concavity of $\bar{\Xi}$ w.r.t. $N$.

It can be seen from Theorem 3 that the optimal $N$ can be obtained in closed-form once $P_{\mathrm{c}}$ is fixed. So the optimal solution of $P_{1}$ can be obtained by a one-dimensional search w.r.t. $P_{\mathrm{c}}$ in the interval of $\left(0, P_{\mathrm{c}}^{\max }\right]$.

\section{Numerical and Simulation Results}

In this section, the Monte Carlo simulations are performed over $10^{5}$ independent trials. We verify the tightness of our analysis and show the optimal number of antennas obtained by our closed-form expression for a given $P_{\mathrm{c}}$. The large-scale fading (path-loss) at distance $d$ is set as $\mathcal{L}(d)=10^{-3.53} d^{-3.76}$ [19]. The distance between the D2D users is set as $10 \mathrm{~m}$. Without loss of 
generality, $K=15$ CUEs and the D2D link are uniformly placed in a circle of radius $200 \mathrm{~m}$ around the BS. The other parameter settings are detailed in Table 1.

Table 1. Simulation Parameters.

\begin{tabular}{cccc}
\hline Parameter & Value & Parameter & Value \\
\hline$\sigma^{2}$ and $\sigma_{d}^{2}$ & $-96 \mathrm{dBm}[19]$ & $P_{\mathrm{d}}$ & $20 \mathrm{dBm}$ \\
\hline$P_{0}$ & $40 \mathrm{dBm}[20]$ & $\gamma_{c}, \gamma_{d}$, and $\tilde{\gamma}_{t h}$ & $10 \mathrm{~dB}$ \\
\hline$r$ & $6 \mathrm{bps} / \mathrm{Hz}$ & $N_{t h}$ & $33[20]$ \\
\hline
\end{tabular}

Figure 2 shows the PDF $f_{\gamma_{c k}}(x)$ obtained by our closed-form approximation and the simulations with $P_{\mathrm{c}}=500 \mathrm{~mW}$ for different number of antennas. A good match can be observed between our closed-form approximation and the simulations.

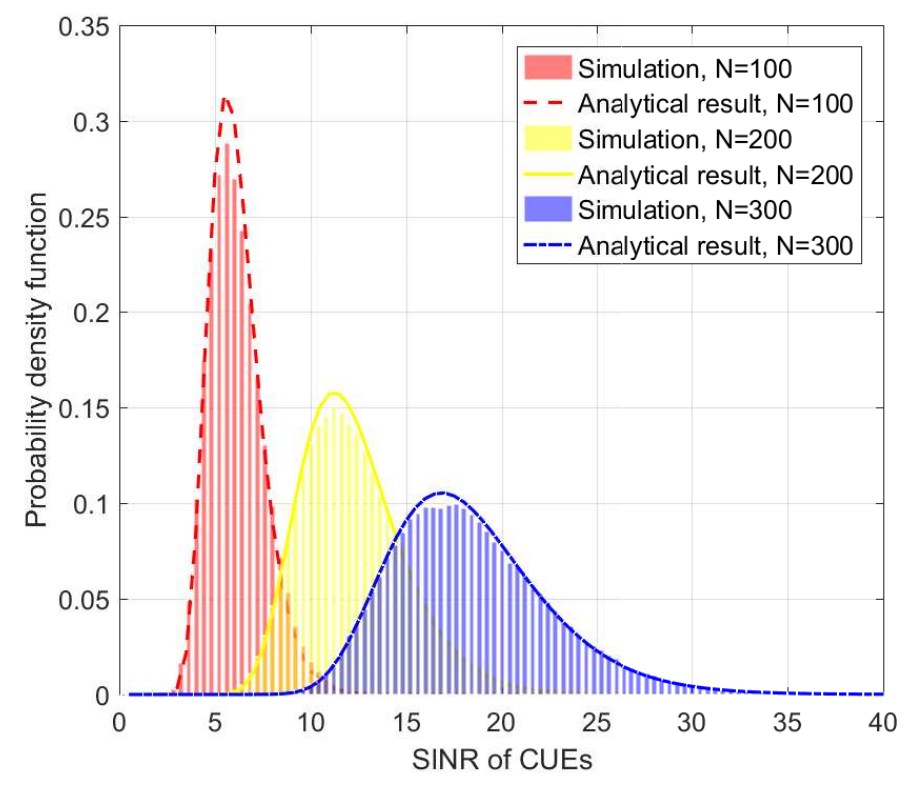

Figure 2. The probability density function of the signal-to-interference-plus-noise ratio (SINR) of cellular user equipments (CUEs), $P_{\mathrm{c}}=500 \mathrm{~mW}$.

Figure 3 plots the achievable rate and the outage probability of CUEs versus the number of antennas. It reveals that the analytical results agree reasonably well with the simulation results. With the increase of the number of BS antennas, the achievable rate can be significantly improved. In addition, the derived lower bound rate is close to the real value. To evaluate the impact of $P_{\mathrm{c}}$ on the performance of massive MIMO system, the achievable rate of CUEs is shown in Figure 4. The achievable rate gets larger and approaches the upper bound with the increase of $P_{\mathrm{c}}$. It can be seen that increasing the transmit power imposes less impacts compared with increasing the number of antennas.

We have $P_{\mathrm{c}}^{\max }=1100 \mathrm{~mW}$ by solving $\mathcal{R}_{d}\left(P_{\mathrm{c}}\right)=r$. Then, the optimal solution of $\mathrm{P} 1$ can be obtained by a one-dimensional search w.r.t. $P_{\mathrm{c}}$ from 0 to $1100 \mathrm{~mW}$. Figure 5. plots the EE for different number of BS antennas and different $P_{\text {cir }}$ for a given $P_{\mathrm{c}}=500 \mathrm{~mW}$. From the plot, we can see that the analytical result is the same as the result obtained by the exhaustive search. As expected, the optimal number of antennas gets smaller with the increase of $P_{\text {cir }}$. 

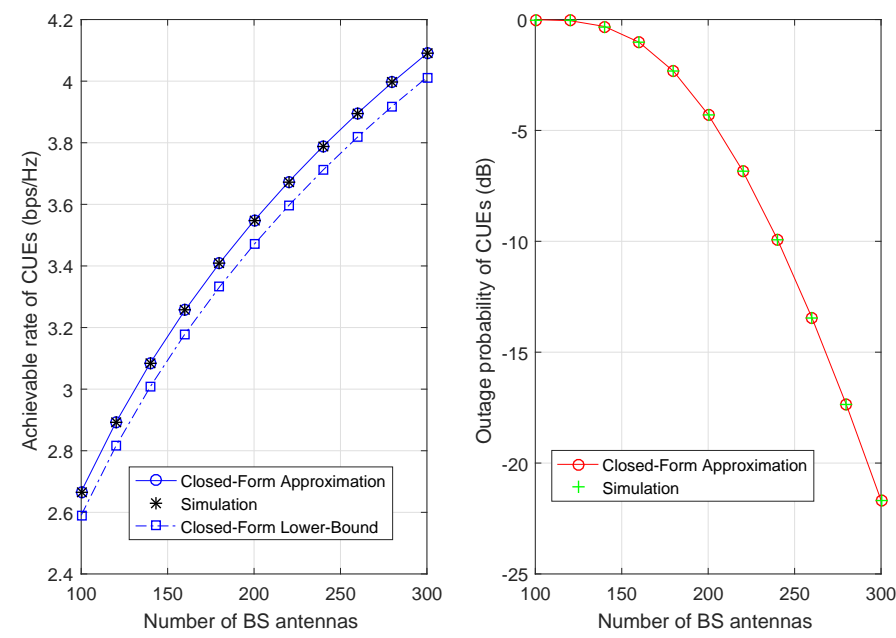

Figure 3. The achievable rate and the outage probability of CUEs vs. the number of base station (BS) antennas, $P_{\mathrm{c}}=500 \mathrm{~mW}$.

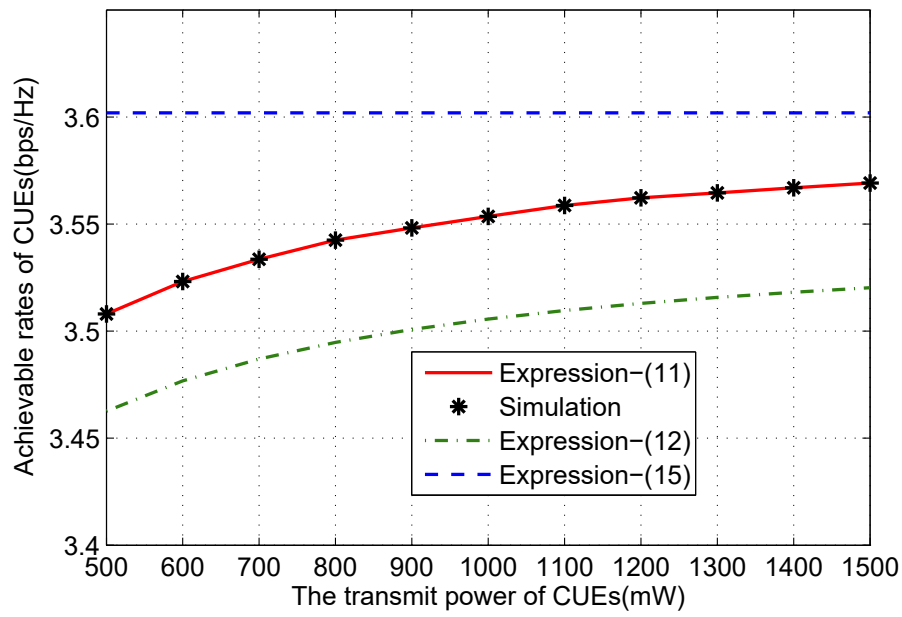

Figure 4. The achievable rate of CUEs vs. the transmit power of CUEs, $N=150$.

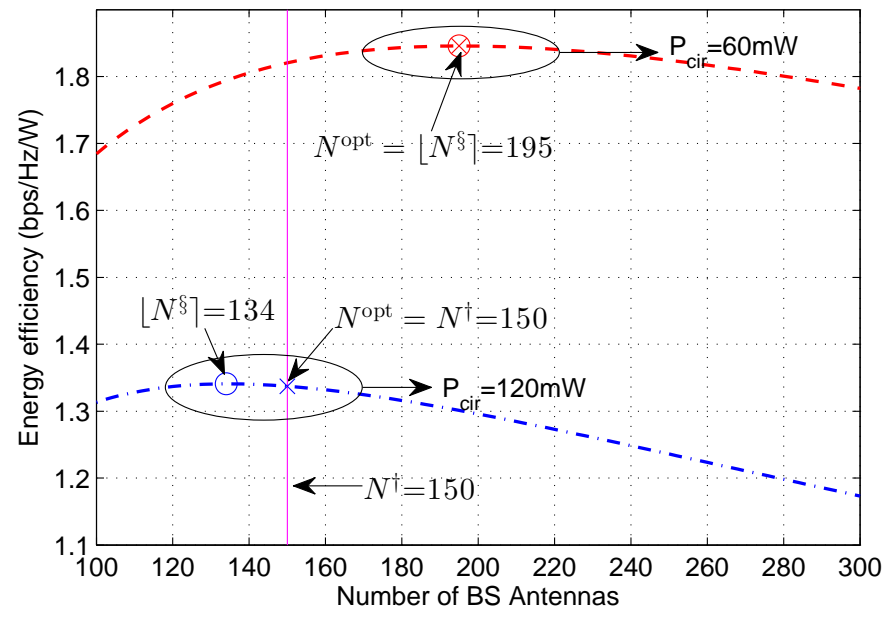

Figure 5. Energy efficiency for different number of BS antennas and different $P_{\text {cir }}, P_{\mathrm{C}}=500 \mathrm{~mW}$. 
Figure 6 compares the maximized EE and the optimal number of antennas obtained by our proposed method and the exhaustive search. As expected, our proposed method achieves the same performance as the optimal exhaustive search with higher complexity.
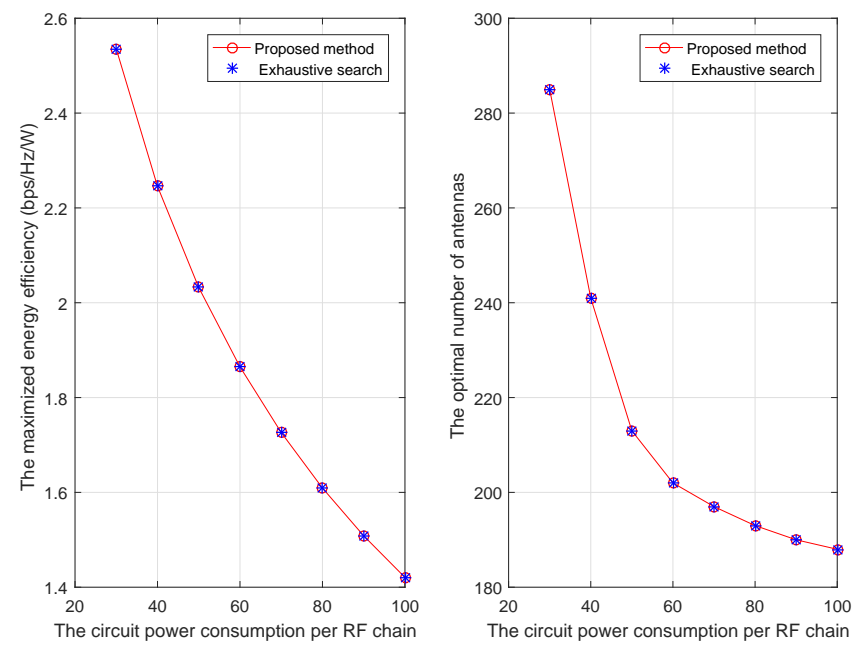

Figure 6. Comparison between our proposed method and the exhaustive search.

\section{Conclusions}

We have derived the accurate closed-form expressions of the outage probability and average achievable rate for a heterogeneous network consisting of an uplink massive MIMO and a D2D link. The influence of the power of CUEs is investigated for the D2D and the CUEs. Constrained by the performance of the D2D link and CUEs, the EE of the massive MIMO system is maximized by jointly optimizing the number of BS antennas and the transmit power of CUEs. Meanwhile, the optimization problem is solved by a one-dimensional search with low complexity. Numerical results show the tightness of our closed-form expressions and reveal the impacts of various parameter settings. In particular, it is interesting to study some extensions of this work similarly to [21-23].

Author Contributions: Xinhua Wang created the main ideas and wrote the paper.

Conflicts of Interest: The authors declare no conflict of interest.

\section{Appendix A. Proof of Theorem 1}

We can rewrite the $n$-th element of $\mathbf{h}_{c k}$ in the polar form, i.e., $\mathbf{h}_{c k}(n)=\left|\mathbf{h}_{c k}(n)\right| e^{i \theta_{n}}$. According to the circular symmetry property, $\mathbf{h}_{c k}^{*}(n) \mathbf{h}_{c j}(n) /\left\|\mathbf{h}_{c k}\right\|$ has the same distribution with $\left|\mathbf{h}_{c k}(n)\right| \mathbf{h}_{c j}(n) /\left\|\mathbf{h}_{c k}\right\|$. Conditioned on $\mathbf{h}_{c k}, \mathbf{h}_{c k}^{*}(n) \mathbf{h}_{c j}(n) /\left\|\mathbf{h}_{c k}\right\| \sim \mathcal{C N}\left(0,\left|\mathbf{h}_{c k}(n)\right| /\left\|\mathbf{h}_{c k}\right\|\right)$. Sum the real part and the imaginary part of the $N$ elements respectively, we have $\Re\left(\frac{\mathbf{h}_{c k}^{H} \mathbf{h}_{c j}}{\left\|\mathbf{h}_{c k}\right\|}\right) \sim \mathcal{N}\left(0, \frac{1}{2}\right)$ and $\Im\left(\frac{\mathbf{h}_{c k}^{H} \mathbf{h}_{c j}}{\left\|\mathbf{h}_{c k}\right\|}\right) \sim \mathcal{N}\left(0, \frac{1}{2}\right)$. In other words, $\frac{\mathbf{h}_{c k}^{H} \mathbf{h}_{c j}}{\left\|\mathbf{h}_{c k}\right\|} \sim \mathcal{C N}(0,1)$ is a random variable independent of $\mathbf{h}_{c k}$. Therefore, $I_{c k}$ is a sum of $K$ independent gamma-distributed random variables. According to ([24] Lemma 3), $I_{c k}$ is a gamma-distributed random variable with the shape $a_{c k}$ and the scale parameter $b_{c k}$, i.e., the PDF of $I_{c k}$ is given as

$$
f_{I_{c k}}(x)=\frac{x^{a_{c k}-1} e^{-x / b_{c k}}}{\left(b_{c k}\right)^{a_{c k}} \Gamma\left(a_{c k}\right)}
$$


According to the law of large numbers, we have $\lim _{N \rightarrow \infty} \frac{\|\mathbf{h}\|^{2}}{N}=1$, so it is straightforward that

$$
S_{c k} \approx P_{c} \beta_{c k} N
$$

Combining (A1) and (A2), the theorem can be proved.

\section{Appendix B. Proof of Theorem 2}

Substitute (A1) and (A2) into (3), we have

$$
\begin{aligned}
\mathcal{R}_{c k} & =\int_{0}^{\infty}\left[\log _{2}\left(\xi_{c k}+I_{c k}\right)-\log _{2}\left(\sigma^{2}+I_{c k}\right)\right] f\left(I_{c k}\right) \mathrm{d} I_{c k} \\
& =\frac{l\left(a_{c k}, b_{c k}, \xi_{c k}\right)-l\left(a_{c k}, b_{c k}, \sigma^{2}\right)}{\left(b_{c k}\right)^{a_{c k}} \Gamma\left(a_{c k}\right) \ln (2)},
\end{aligned}
$$

where $l\left(t_{1}, t_{2}, t_{3}\right)=\int_{0}^{\infty} x^{\left(t_{1}-1\right)} e^{-\frac{x}{t_{2}}} \ln \left(t_{3}+x\right) \mathrm{d} x$. The integrals $l\left(a_{c k}, b_{c k}, \xi_{c k}\right)$ and $l\left(a_{c k}, b_{c k}, \sigma^{2}\right)$ cannot be solved according to the ([18] (Equation (4.222.8)) because $a_{c k}$ and $b_{c k}$ are positive but not necessarily integers. However, $l\left(a_{c k}, b_{c k}, \xi_{c k}\right)$ can be derived as follows in closed-form using the property of Meijer's G-function,

$$
\begin{aligned}
& l\left(a_{c k}, b_{c k}, \xi_{c k}\right)=\int_{0}^{\infty} x^{\left(a_{c k}-1\right)} e^{-\frac{x}{b_{c k}}} \ln \left(\xi_{c k}\right) \mathrm{d} x+\int_{0}^{\infty} x^{\left(a_{c k}-1\right)} e^{-\frac{x}{b_{c k}}} \ln \left(1+x / \xi_{c k}\right) \mathrm{d} x \\
& \stackrel{(a)}{=} \ln \left(\xi_{c k}\right)\left(b_{c k}\right)^{a_{c k}} \Gamma\left(a_{c k}\right)+\int_{0}^{\infty} x^{\left(a_{c k}-1\right)} e^{-\frac{x}{b_{c k}}} G_{2,2}^{1,2}\left(\frac{x}{\xi_{c k}} \mid \begin{array}{l}
1,1 \\
1,0
\end{array}\right) \mathrm{d} x \\
& \stackrel{(b)}{=}\left(b_{c k}\right)^{a_{c k}}\left[\mathcal{J}\left(a_{c k}, b_{c k} / \xi_{c k}\right)+\ln \left(\xi_{c k}\right) \Gamma\left(a_{c k}\right)\right] .
\end{aligned}
$$

Step (a) is obtained by representing $\ln \left(1+x / \xi_{c k}\right)$ as the Meijer's G-function, and step (b) is derived according to ([25] Equation (44)). Similarly to (A4), we can derive $l\left(a_{c k}, b_{c k}, \sigma^{2}\right)$, and concludes the proof.

\section{References}

1. Larsson, E. G.; Edfors, O.; Tufvesson, F.; Marzetta, T.L. Massive MIMO for next generation wireless systems. IEEE Commun. Mag. 2014, 52, 186-195.

2. Björnson, E.; Larsson, E.G.; and Marzetta, T.L. Massive MIMO: Ten myths and one critical question. IEEE Commun. Mag. 2016, 54, 114-123.

3. Ngo, H.Q.; Larsson, E.G.; Marzetta, T.L. Energy and spectral efficiency of very large multiuser MIMO systems. IEEE Trans. Commun. 2013, 61, 1436-1449.

4. Hong, Y.; Marzetta, T.L. Performance of conjugate and zero-forcing beamforming in large-scale antenna systems. IEEE J. Sel. Areas Commun. 2013, 31, 172-179.

5. Nguyen, H.D.; Sun, S. Massive MIMO versus small-cell systems: Spectral and energy efficiency comparison. In Proceedings of the 2016 IEEE International Conference on Communications, Kuala Lumpur, Malaysia, 22-27 May 2016; pp. 1-6.

6. Masini, B.M.; Conti, A. Adaptive TORC detection for MC-CDMA wireless systems. IEEE Trans. Commun. 2009, 57, 3460-3471.

7. Masini, B.M. The impact of combined equalization on the performance of MC-CDMA systems. J. Commun. 2008, 3, 31-39.

8. Wen, C.K.; Jin, S.; Wong, K.K. On the sum-rate of multiuser MIMO uplink channels with jointly-correlated Rician fading. IEEE Trans. Commun. 2011, 59, 2883-2895.

9. Wei, L.; Hu, R. Q.; Qian, Y.; Wu, G. Enable Device-to-Device communications underlaying cellular networks: Challenges and research aspects. IEEE Commun. Mag. 2014, 52, 90-96. 
10. Asadi, A.; Wang, Q.; Mancuso, V. A survey on Device-to-Device communication in cellular networks. IEEE Commun. Surv. Tutor. 2016, 16, 1801-1819.

11. Doppler, K.; Rinne, M.; Wijting, C.; Ribeiro, C.B.; Hugl, K. Device-to-Device communication as an underlay to LTE-advanced networks. IEEE Commun. Mag. 2009, 47, 42-49.

12. Min, H.; Lee, J.; Park, S.; Hong, D. Capacity enhancement using an interference limited area for Device-to-Device uplink underlaying cellular networks. IEEE Trans. Wireless Commun. 2011, 10, 3995-4000.

13. Bazzi, A.; Masini, B.M.; Zanella, A. Performance analysis of V2V beaconing using LTE in direct mode with full duplex radios. IEEE Commun. Lett. 2015, 4, 685-688.

14. Palombara, C.L.; Tralli, V.; Masini, B.M.; Conti, A. Relay-assisted diversity communications. IEEE Trans. Veh. Technol. 2013, 62, 415-421.

15. Lin, X.; Heath, R.W.; Andrews, J.G. The interplay between massive MIMO and underlaid D2D networking. IEEE Trans. Wirel. Commun. 2015, 14, 3337-3351.

16. Amin, B. S.; Ramadan, Y. R.; Ibrahim, A. S.; Ismail, M. H. Power allocation for Device-to-Device communication underlaying massive MIMO multicasting networks. In Proceedings of the Wireless Communications and Networking Conference (WCNC), New Orleans, LA, USA, 9-12 March 2015; pp. 1219-1224.

17. Shalmashi, S.; Bjornson, E.; Kountouris, M.; Sung, K.W. Energy efficiency and sum rate when massive MIMO meets Device-to-Device communication. In Proceedings of the 2015 IEEE International Conference on Communication Workshop (ICCW), Londin, UK, 8-12 June 2015; pp. 627-632.

18. Gradshteyn, I.S.; Ryzhik, I.M. Table of Integrals, Series, and Products, 6th ed.; Academic: New York, NY, USA, 1994.

19. Bj̈̈nson, E.; Sanguinetti, L.; Hoydis, J.; Debbah, M. Optimal design of energy-efficient multi-user MIMO systems: Is massive MIMO the answer? IEEE Trans. Wirel. Commun. 2015, 14, 3059-3075.

20. Ng, D.W.K.; Lo, E.S.; Schober, R. Energy-efficient resource allocation in OFDMA systems with large numbers of base station antennas. IEEE Trans. Commun. 2009, 11, 3292-3304.

21. Emmanouil, S.; Traganitis, A.; Ephremides, A. Rate region and power considerations in a simple $2 \times 2$ interference channel. In Proceedings of the IEEE Information Theory Workshop on Networking and Information Theory, University of Thessaly, Volos, Greece, 10-12 June 2009; pp. 296-300.

22. Spanakis, E.; Sakkalis, V.; Marias, K.; Traganitis, A. Cross Layer Interference Management in Wireless Biomedical Networks. Entropy 2014, 16, 2085-2104.

23. Pefkianakis, I.; Hu, Y.; Wong, S.; Yang, H.; Lu, S. MIMO rate adaptation in 802.11n wireless networks. In Proceedings of the ACM International Conference on Mobile Computing and Networking, Chicago, IL, US, 20-24 September 2010; Volume 18, pp. 257-268.

24. Hosseini, K.; Yu, W.; Adve, R. Large-scale MIMO versus network MIMO for multicell interference mitigation. IEEE J. Sel. Top. Signal Process. 2014, 8, 930-941.

25. Zhong, C.; Wong, K.K.; Jin, S. Capacity bounds for MIMO nakagami- $m$ fading channels. IEEE Trans. Signal Process. 2009, 57, 3613-3623.

(C) 2017 by the authors. Licensee MDPI, Basel, Switzerland. This article is an open access article distributed under the terms and conditions of the Creative Commons Attribution (CC BY) license (http:// creativecommons.org/licenses/by/4.0/). 